\title{
Correlation of Clinical Changes with Regard to Thyroxine Replacement Therapy in Hypothyroid Patients - Focusing on the Change of Renal Function
}

\author{
Kuei-Mei Chou $^{b}$ Sherry Yueh-Hsia Chiu ${ }^{a} \quad$ Chih-Huang Chen ${ }^{b} \quad$ Ning-I Yang ${ }^{c}$ \\ Be-Yi Huang ${ }^{b}$ Chiao-Yin Sun ${ }^{d}$ \\ ${ }^{a}$ Department of Health Care Management, Chang Gung University, Tao-Yuan, and Divisions of ${ }^{b}$ Endocrinology \\ and Metabolism, 'C Cardiology, and d Nephrology, Department of Internal Medicine, Chang Gung Memorial Hospital, \\ Keelung, Taiwan, ROC
}

\section{Key Words}

Hypothyroidism • Renal function • Ejection fraction of left

ventricle $\cdot$ Renal vascular function

\begin{abstract}
Thyroid dysfunction has an important role in renal insufficiency. The aim of the study was to correlate the change of renal function with other clinical factors after thyroxine therapy in hypothyroid patients. A prospective study was designed and 30 hypothyroid patients were included. All study subjects received $0.15-0.2 \mathrm{mg} /$ day thyroxine for 12 weeks. Diastolic blood pressure and serum levels of creatine phosphokinase (CPK) and myoglobulin decreased significantly after thyroxine therapy. Serum creatinine decreased $(0.87 \pm$ 0.22 vs. $0.70 \pm 0.17 \mathrm{mg} / \mathrm{dl}, \mathrm{p}<0.001)$ and estimated glomerular filtration rate (eGFR) increased significantly (82.06 \pm 31.08 vs. $100.31 \pm 31.79 \mathrm{ml} / \mathrm{min} / 1.73 \mathrm{~m}^{2} ; \mathrm{p}<0.001$ ) after thyroxine replacement. Left ventricular ejection fraction (LVEF) was significantly increased after thyroxine replacement $(64.47 \pm 11.94$ vs. $72.40 \pm 13.89 \%, p=0.026)$. No significant vascular functional changes of peripheral (pulse wave velocity) and renal interlobar arteries (pulsatility index and resistance index) were noted. The change of eGFR significantly
\end{abstract}

correlated with the changes of serum-free $T_{4}\left(\mathrm{fT}_{4}\right), \mathrm{CPK}$, myoglobulin and LVEF. The correlation between the change of eGFR and thyroid-stimulating hormone (TSH) level was not significant. In conclusion, the GFR of hypothyroid patients increased significantly after thyroxine replacement. The change of GFR was significantly correlated with the changes of $\mathrm{fT}_{4}, \mathrm{CPK}$, myoglobulin and LVEF, but not with TSH.

Copyright $\odot 2011$ S. Karger AG, Basel

\section{Introduction}

The evaluation of thyroid function in systemic illness remains complex because the changes occur at all levels of the pituitary-thyroid axis [1]. Thyroid hormones have an important role in kidney growth and in the maintenance of many of its functions [2]. Hypothyroidism was reported to be associated with albuminuria and decreased glomerular filtration rate (GFR) $[3,4]$. The association of this renal dysfunction may be due to decrease in resorption in the proximal tubules [5], impairment in the concentration and diluting capacities of the distal tubules [6], a decrease in urinary excretion [7], and a decrease in renal blood flow [8]. Only a short period of hypothyroidism is

\section{KARGER}

Fax +4161306 1234 E-Mail karger@karger.ch www.karger.com

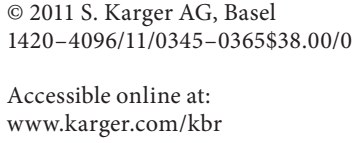

Chiao-Yin Sun, MD

Division of Nephrology, Chang Gung Memorial Hospital

222 Mai-Chin Road, Keelung, Taiwan (ROC)

Tel. +886 22431 3131, ext. 3169

E-Mail fish3970@gmail.com 
required to develop elevated serum creatinine levels, and renal function improves significantly after treatment for hypothyroidism [9].

Thyroid dysfunction is also associated with marked alteration in cardiovascular function [3]. In hypothyroidism, myocardial contractility, cardiac output and oxygen consumption are decreased [10], whereas peripheral vascular resistance is increased [8]. The cause of decreased renal plasma blood flow and GFR observed is believed to be principally due to the generalized hypodynamic state of the circulatory system and the absence of inotropic and chronotropic effects of the thyroid hormones in hypothyroidism [11].

Muscular symptoms with creatine phosphokinase (CPK) elevations were found in more than $90 \%$ of cases. Hypothyroidism induces renal dysfunction and especially decreased GFR was due in part to some degree of myopathy [12]. Hypothyroidism might reduce glycolysis and oxidative phosphorylations and thus reduce adenosine triphosphate concentrations. This impairment of muscle function and muscle energy metabolism can lead to an increase of CPK and myoglobulin release from skeletal muscle and may be elevated in $70-90 \%$ of hypothyroid patients $[13,14]$. Elevation of CPK and myoglobulin, which are toxic for renal tubules and could cause renal tubule obstruction, will result in harmful side effects of renal damage $[12,14]$.

It has been reported that there is a frequent association between hypothyroidism and decreased GFR in patients with chronic kidney disease and preserved renal function $[15,16]$. Previous studies have shown that most patients recovered renal function 6 months after correction of hypothyroidism $[4,17,18]$. However, the changes of renal function in hypothyroidism patients after a short duration of thyroxine therapy and the linkage between renal function and other clinical factors have rarely been studied. In this study, we prospectively analyzed the changes of renal function after short-term thyroxine therapy in hypothyroid patients, and analyzed the correlation between renal and thyroid function, cardiovascular function and myopathy.

\section{Subjects and Methods}

A prospective study was designed. The study subjects $(\mathrm{n}=30)$ and study flow are plotted in figure 1.30 hypothyroid patients were examined as study subjects, which included 24 hypothyroid patients secondary to withdrawal from thyroxine therapy 4 weeks prior to the scheduled radioiodine procedure for thyroid cancer and 6 patients with untreated primary autoimmune hypothyroid-

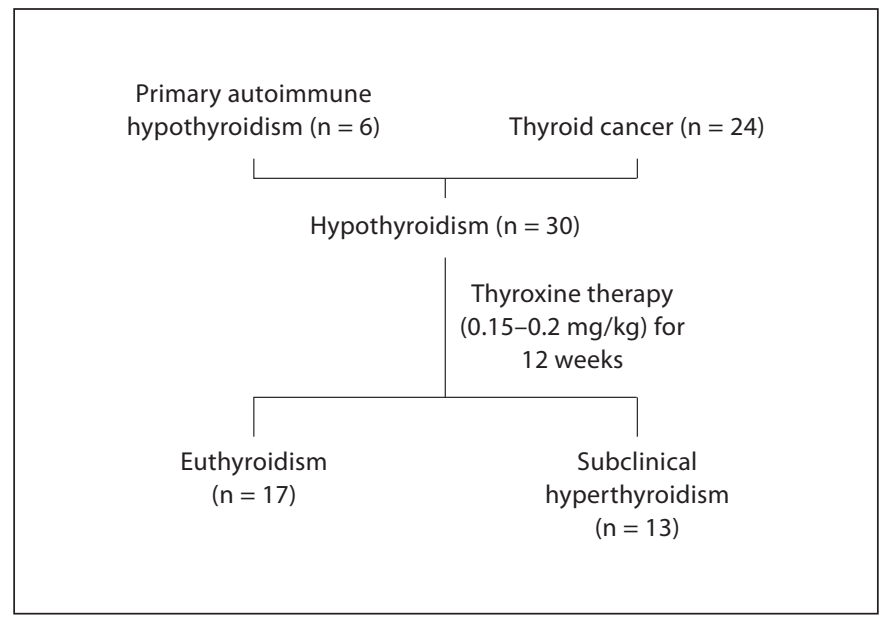

Fig. 1. Study subjects and study flow.

ism. There were 20 patients with papillary cancer and 4 patients with follicular cancer among the patients with thyroid cancer, and 3 patients had local neck lymph node metastasis. No patients had distant metastasis. The patients with thyroid cancer all had surgical tumor resection before taking radiotherapy, and received no thyroxine replacement therapy or other chemical therapy during the 4-week withdrawal period. All patients presented with the classical signs and symptoms of thyroid deficiency, low to undetectable serum-free $\mathrm{T}_{4}\left(\mathrm{fT}_{4}\right)$ levels and markedly elevated serum thyroid-stimulating hormone (TSH) levels. Persons with pregnancy, infection disease, and terminal stage of kidney disease were not included. All study subjects received $0.15-0.2 \mathrm{mg}$ /day thyroxine for 12 weeks.

Thyroid function tests were performed using electrochemiluminescence assay. Renal function, serum CPK and myoglobulin were measured in all participants at the beginning and end of the study. Estimated GFRs (eGFR) were calculated with MDRD equation $\left[\mathrm{eGFR}\left(\mathrm{ml} / \mathrm{min} / 1.73 \mathrm{~m}^{2}\right)=1.86 \times(\mathrm{Scr})^{-1.154} \times(\text { Age })^{-0.23} \times\right.$ (0.742 if female), Scr (serum creatinine, $\mathrm{mg} / \mathrm{dl}$ )]. Spot urine was used for measuring the amount of albuminuria. Urine albumin excretion was calculated with the urine albumin/creatinine ratio.

Standard two-dimensional echocardiogram of the heart (transthoracic iE33 ultrasound system; Philips Medical System, Best, The Netherlands) was obtained in the left lateral position. End-diastolic volume (EDV), end-systolic volume (ESV), ejection fraction (EF) and posterior wall thickness (PWd) of the left ventricle (LV) were determined from the parasternal long axis view using the Teichholz method (fig. 2a). The measurements were carried out by a single cardiologist and interpreted according to recommendations of the American Society of Echocardiography.

Peripheral arterial stiffness was evaluated by brachial-ankle pulse wave velocity (PWV). The brachial-ankle PWVs (pulse wave machine, Colin VP-2000/1000 Vascular Profiling System; Colin Corp., Japan) was measured after resting at least $15 \mathrm{~min}$. The sampling time was $10 \mathrm{~s}$ with automatic gain analysis and quality adjustment. Bilateral brachial-ankle PWVs were measured and the mean value of bilateral brachial-ankle PWVs was used for static analysis. 


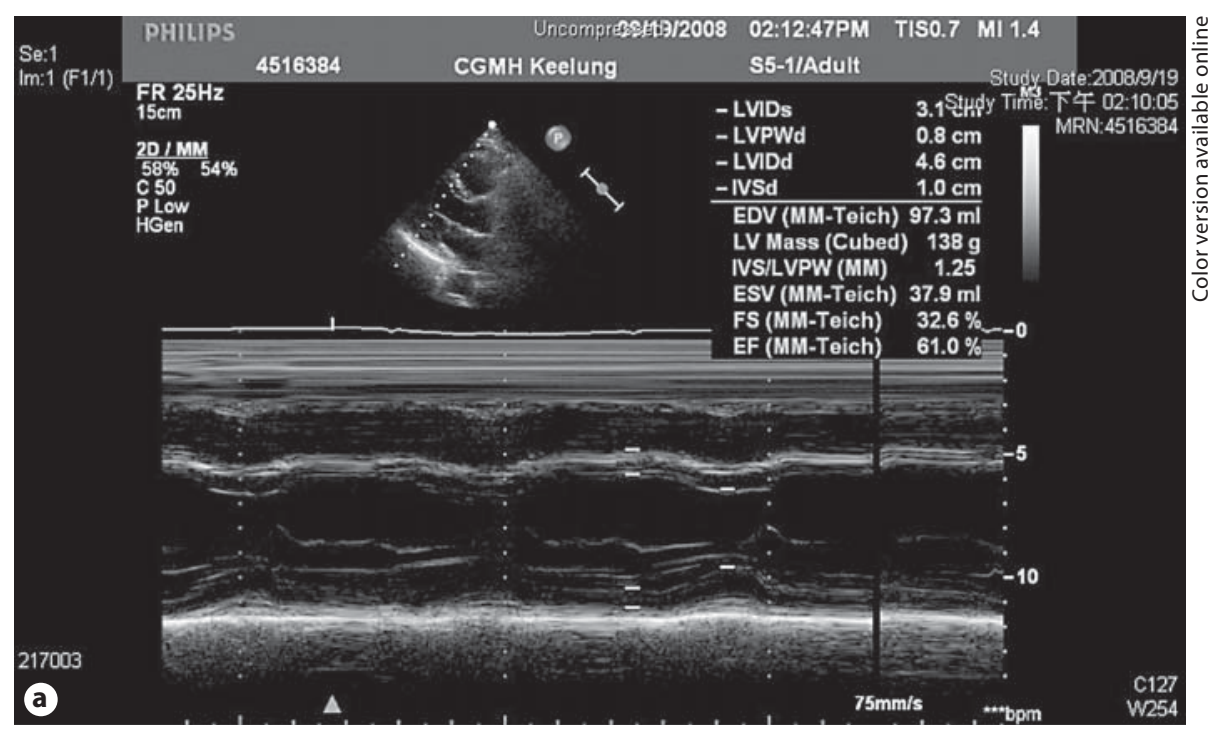

Fig. 2. Representative cases of two-dimensional echocardiography (a) and Doppler flow echogram (b) of kidney.

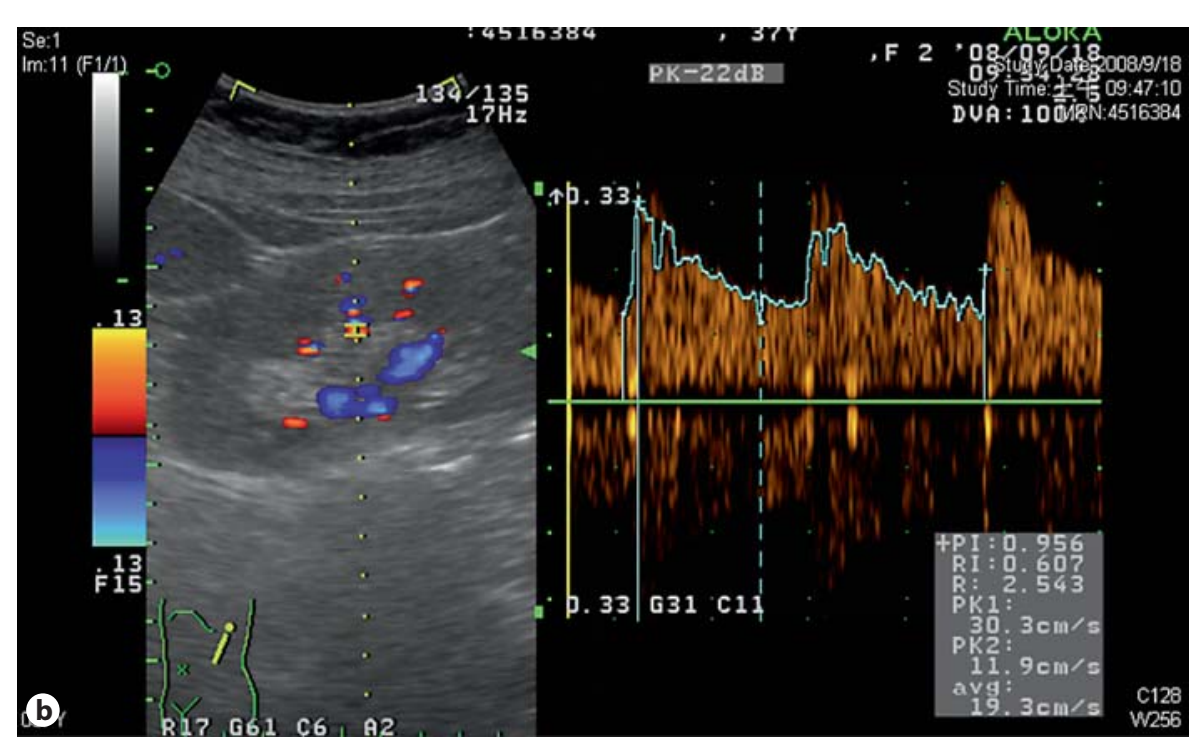

Doppler flow echography of bilateral kidneys performed by a single nephrologist was done for calculating pulsatility index (PI) and resistance index (RI) with an Aloka SSD-5000 machine. Intrarenal Doppler spectra of the interlobar arteries were obtained at three representative locations of the bilateral kidneys (fig. 2b). RI was calculated as (peak systolic frequency shift - minimum diastolic frequency shift)/peak systolic frequency shift, and PI as (peak systolic frequency shift - minimum diastolic frequency shift)/mean frequency shift. The average RI and PI of the bilateral kidneys were computed to yield overall RI and PI values. The physicians performing the clinical examinations were blind to the study subjects.

Data were expressed as means \pm SD. The data of different study groups were compared with Student's t test, and the correlation between variables was analyzed by Pearson's correlation. $\mathrm{p}$ values of $<0.05$ were considered statistically significant.

Change of GRF in Hypothyroidism after Thyroxine Replacement Therapy

\section{Results}

A decrease of GFR was noted after a short-lived thyroxine withdrawal for 4 weeks in thyroidectomized patients $\left(108 \pm 43.43\right.$ vs. $\left.82.06 \pm 31.08 \mathrm{ml} / \mathrm{min} / 1.73 \mathrm{~m}^{2}\right)$. All patients $(\mathrm{n}=30, \mathrm{M} / \mathrm{F}$ 5/25) were treated with 3-month thyroxine replacement (thyroxine dose from 0.15 to 0.2 $\mathrm{mg} /$ day). The average age of study subjects was $51 \pm 11.4$ years. There were 17 patients obtaining euthyroid status and 13 patients obtaining subclinical hyperthyroidism (TSH $<0.2 \mathrm{mU} / \mathrm{l})$. The clinical data before and after thyroxine replacement are shown in table 1 . $\mathrm{fT}_{4}$ was increased and TSH decreased significantly after thyroxine replacement therapy. BMI, systolic blood pressure (SBP) 
Table 1. Clinical parameters before and after thyroxine therapy

\begin{tabular}{|c|c|c|c|c|}
\hline Variable & Before $(n=30)$ & $\operatorname{After}(\mathrm{n}=30)$ & Difference (after-before) & $\mathrm{p}$ value \\
\hline BMI & $24.44 \pm 4.18$ & $24.72 \pm 4.43$ & $-0.55 \pm 3.76$ & 0.768 \\
\hline $\mathrm{SBP}, \mathrm{mm} \mathrm{Hg}$ & $125.00 \pm 17.32$ & $127.00 \pm 13.33$ & $2.23 \pm 21.86$ & 0.666 \\
\hline $\mathrm{DBP}, \mathrm{mm} \mathrm{Hg}$ & $76.55 \pm 11.67$ & $71.25 \pm 9.20$ & $-5.10 \pm 11.13$ & 0.020 \\
\hline Heart rate, bpm & $74.66 \pm 7.70$ & $78.14 \pm 8.25$ & $3.48 \pm 9.74$ & 0.065 \\
\hline $\mathrm{TSH}, \mathrm{mU} / \mathrm{l}$ & $73.58 \pm 61.91$ & $0.79 \pm 1.13$ & $-72.79 \pm 61.88$ & $<0.001$ \\
\hline $\mathrm{fT}_{4}, \mathrm{pmol} / \mathrm{l}$ & $0.31 \pm 0.14$ & $1.58 \pm 0.25$ & $1.27 \pm 0.29$ & $<0.001$ \\
\hline BUN, mg/dl & $11.71 \pm 4.20$ & $12.89 \pm 3.73$ & $1.18 \pm 4.05$ & 0.135 \\
\hline Creatinine, mg/dl & $0.87 \pm 0.22$ & $0.70 \pm 0.17$ & $-0.17 \pm 0.10$ & $<0.001$ \\
\hline $\mathrm{eGFR}, \mathrm{ml} / \mathrm{min} / 1.73 \mathrm{~m}^{2}$ & $82.06 \pm 31.08$ & $100.31 \pm 31.79$ & $18.26 \pm 12.56$ & $<0.001$ \\
\hline Albuminuria, mg/dl & $43.86 \pm 124.67$ & $13.58 \pm 2.08$ & $-30.28 \pm 113.22$ & 0.168 \\
\hline $\mathrm{CPK}, \mathrm{mg} / \mathrm{dl}$ & $262.11 \pm 149.05$ & $109.56 \pm 71.55$ & $-152.56 \pm 129.57$ & $<0.001$ \\
\hline Myoglobulin, mg/dl & $32.84 \pm 16.23$ & $21.07 \pm 7.48$ & $-11.76 \pm 13.67$ & $<0.001$ \\
\hline LVEDV, $\mathrm{ml}$ & $100.01 \pm 22.06$ & $101.06 \pm 27.00$ & $1.01 \pm 21.02$ & 0.893 \\
\hline LVESV, ml & $33.77 \pm 15.72$ & $23.60 \pm 6.68$ & $-9.83 \pm 14.21$ & 0.002 \\
\hline LVEF, \% & $64.47 \pm 11.94$ & $72.40 \pm 13.89$ & $7.93 \pm 17.81$ & 0.026 \\
\hline LVPWd, \% & $0.85 \pm 0.021$ & $0.83 \pm 0.020$ & $0.00 \pm 0.01$ & 0.840 \\
\hline Average $\mathrm{PWV}, \mathrm{cm} / \mathrm{s}$ & $1,406.71 \pm 232.50$ & $1,358.50 \pm 235.72$ & $-48.21 \pm 141.81$ & 0.083 \\
\hline Average kidney PI & $1.19 \pm 0.24$ & $1.25 \pm 0.20$ & $0.06 \pm 0.29$ & 0.274 \\
\hline Average kidney RI & $0.69 \pm 0.09$ & $0.71 \pm 0.07$ & $0.01 \pm 0.11$ & 0.536 \\
\hline
\end{tabular}

LVEDV = End-diastolic volume of the left ventricle; LVESV= end-systolic volume of the left ventricle; LVPWd = posterior wall thickness of the left ventricle; $\mathrm{LVEF}=$ ejection fraction of the left ventricle; PWV = pulse wave velocity; PI = pulsatility index; RI = resistance index.

and heart rate did not significantly change. Diastolic blood pressure (DBP) decreased after thyroxine treatment $(76.55 \pm 11.67$ vs. $71.25 \pm 9.20 \mathrm{~mm} \mathrm{Hg}, \mathrm{p}=0.020)$. The serum marker for myopathy, CPK and myoglobulin, decreased significantly after thyroxine therapy.

The amount of albuminuria did not significantly differ after thyroxine therapy $(43.86 \pm 124.67$ vs. $13.58 \pm$ $2.08 \mathrm{mg} / \mathrm{dl}, \mathrm{p}=0.168)$. However, there were 4 patients with microalbuminuria and 2 patients with macroalbuminuria before thyroxine therapy. After thyroxine replacement, 3 microalbuminuria patients became normal and 2 macroalbuminuria patients improved to microalbuminuria after thyroxine therapy. The serum creatinine decreased $(0.87 \pm 0.22$ vs. $0.70 \pm 0.17 \mathrm{mg} / \mathrm{dl}, \mathrm{p}<0.001)$ and eGFR increased significantly $(82.06 \pm 31.08$ vs. $\left.100.31 \pm 31.79 \mathrm{ml} / \mathrm{min} / 1.73 \mathrm{~m}^{2}, \mathrm{p}<0.001\right)$ after thyroxine replacement. However, the serum BUN level did not differ before or after therapy (table 1).

End-systolic volume of the left ventricle (LVESV) significantly decreased after thyroxine therapy (33.77 \pm 15.72 vs. $23.60 \pm 6.68 \mathrm{ml}, \mathrm{p}=0.002$ ). No significant changes of end-systolic volume of the left ventricle (LVEDV) and posterior wall thickness of the left ventricle (LVPWd) were noted after thyroxine replacement.
Left ventricular ejection fraction (LVEF) was significantly increased after thyroxine replacement (64.47 \pm 11.94 vs. $72.40 \pm 13.89 \%, p=0.026)$. Among the study subjects, 1 patient had severely impaired cardiac function (LVEF 12.1\%) before thyroxine therapy. After 3 months of thyroxine therapy, this patient's LVEF increased to $67.6 \%$. The average brachial-ankle PWV did not significantly change after therapy. PI and RI of renal interlobar arteries did not significantly change either (table 1).

The results of Pearson's correlation between the changes of thyroid function and other clinical parameters are summarized in table 2 . There was no significant correlation between the changes of $\mathrm{fT}_{4}$ and TSH levels (Pearson's correlation $-0.103, p=0.586)$. The changes of eGFR, CPK, myoglobulin, and LVEF were significantly correlated with the changes of $\mathrm{fT}_{4}$, and the correlation was not significant with the change of TSH. The change of DBP was not significantly correlated with $\mathrm{fT}_{4}$ or TSH.

The correlations between the changes of GFR and other clinical parameters were plotted in figure 3. Pearson's correlation analysis results showed that the change of eGFR was significantly correlated with the changes of $\mathrm{fT}_{4}, \mathrm{CPK}$, myoglobulin and LVEF (table 3). Even DBP and 
Table 2. Results of Pearson's correlation between the change of thyroid function and other clinical parameters $(n=30)$; the changes of eGFR, CPK, myoglobulin, and LVEF were significantly correlated with the changes of $\mathrm{fT}_{4}$

\begin{tabular}{|c|c|c|}
\hline & Pearson's correlation (r) & $\mathrm{p}$ \\
\hline \multicolumn{3}{|c|}{ Pearson's correlation with $\mathrm{fT}_{4}$} \\
\hline $\mathrm{DBP}$ & 0.119 & 0.532 \\
\hline eGFR & 0.755 & $<0.001$ \\
\hline CPK & 0.369 & 0.045 \\
\hline Myoglobulin & 0.684 & $<0.001$ \\
\hline LVEF & 0.693 & $<0.001$ \\
\hline \multicolumn{3}{|c|}{ Pearson's correlation with TSH } \\
\hline DBP & -0.030 & 0.876 \\
\hline eGFR & -0.221 & 0.241 \\
\hline $\mathrm{CPK}$ & -0.044 & 0.816 \\
\hline Myoglobulin & -0.002 & 0.992 \\
\hline LVEF & 0.099 & 0.604 \\
\hline
\end{tabular}

Table 3. Results of Pearson's correlation between the change of eGFR and other clinical parameters $(n=30)$; the change of eGFR was significantly correlated with the changes of $\mathrm{fT}_{4}, \mathrm{CPK}$, myoglobulin and LVEF

\begin{tabular}{lrr}
\hline & Pearson's correlation (r) & \multicolumn{1}{c}{$\mathrm{p}$} \\
\hline $\mathrm{DBP}$ & 0.192 & 0.310 \\
$\mathrm{fT}_{4}$ & 0.755 & $<0.001$ \\
$\mathrm{TSH}$ & -0.221 & 0.241 \\
$\mathrm{CPK}$ & 0.372 & 0.043 \\
Myoglobulin & 0.684 & $<0.001$ \\
LVEF & 0.665 & $<0.001$ \\
\hline
\end{tabular}

TSH level significantly changed after thyroxine therapy, and the changes of DBP and TSH did not significantly correlate with the change of eGFR.

\section{Discussion}

Although thyroid dysfunction can play an important role in renal insufficiency $[11,15,19]$, a limited number of studies have linked the change of renal dysfunction with the other clinical changes in hypothyroid patients after thyroxine replacement. This study reveals several interesting observations. A short course of thyroxine replacement therapy could significantly increase GFR in hypothyroidism patients. The change of GFR was signif- icantly correlated with the change of $\mathrm{fT}_{4}$, but not the change of TSH. Our results suggested that the pathophysiological effect of TSH on renal function in the thyroid dysfunction status might be minimal.

After thyroxine replacement, LVEF was significantly increased in our study. Thyroid hormone is an important regulator of cardiac function and cardiovascular hemodynamics. The physiologically active form of thyroid hormone binds to nuclear receptor proteins and mediates the expression of several important cardiac genes, inducing transcription of the positively regulated genes including $\alpha$-myosin heavy chain and the sarcoplasmic reticulum calcium ATPase. Previous studies had showed that hypothyroidism could depress myocardial contractile function and hemodynamics, and it was reversible by substitution with thyroid hormones [20, 21]. Our results suggested that thyroxine replacement could significantly increase contractility of LV in hypothyroidism patients with normal baseline LV function. In addition, our data suggested that no significant effect of thyroid replacement in the diastolic function and myocardium thickness of LV. Our study showed that the changes of LVEF correlated well with the change of $\mathrm{fT}_{4}$, but not TSH level in our study population. A cross-sectional study, the Framingham Heart Study [22], also showed that TSH was not related to LV systolic function in men. The improvement of cardiac function after thyroxine therapy was considered as an important factor for the increased GFR in heart failure patients $[23,24]$. We also demonstrated that the change of LVEF was significantly correlated with the change of GFR along the thyroxine replacement course clinically. Our results suggested that increased LVEF might have an important role in improving GFR in hypothyroidism patients with normal baseline heart function.

Elevated myoglobulin in hypothyroid myopathy could cause renal tubule obstruction, and CPK and myoglobulin were considered to be toxic to renal tubules $[13,14]$. Our study revealed that thyroxine replacement could significantly decrease the CPK and myoglobulin levels in hypothyroidism patients. In addition, the changes of myoglobulin and CPK along with the thyroxine replacement course were significantly correlated with the change of GFR. Based on our results, it was suggested that increased CPK and myoglobulin levels in hypothyroidism patients had significant roles in reducing renal function.

Thyroid hormones play a direct role in renal physiology such as water excretion, tubular transport of sodium, kidney dopaminergic system and renin-angiotensin activity [25]. However, the physiological effect of TSH on the renal function was not defined. Our study showed 

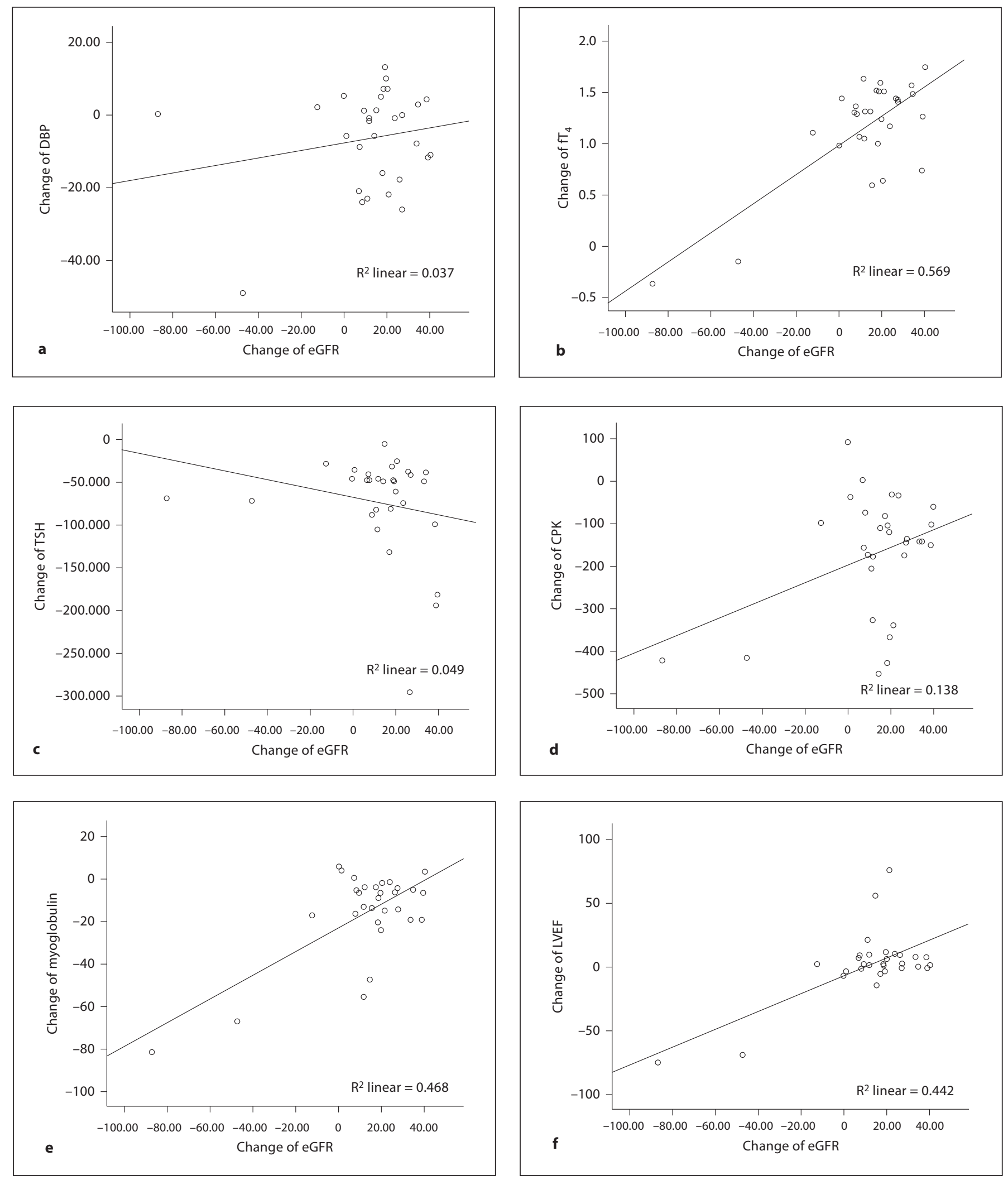

Fig. 3. Plots for the correlation between the changes of eGFR and other clinical parameters $(\mathrm{n}=30)$ : $(\mathbf{a}) \mathrm{DBP}$, (b) $\mathrm{fT}_{4}$, (c) TSH, (d) CPK, (e) myoglobulin, and (f) LVEF. 
that the change of GFR correlated well with the change of $\mathrm{fT}_{4}$ level in hypothyroidism patients after thyroxine replacement. Although the TSH level reflexes the status of thyroid function clinically, the change of GFR was not correlated with the change of TSH level in our study subjects. The other clinical parameters such as myoglobulin, CPK and LVEF also had significant clinical association with the change of $\mathrm{fT}_{4}$ level, but not TSH. Because of the lack of a clinical correlation between TSH and other clinical parameters, it was suggested that TSH might not be a good clinical indicator to monitor hypothyroid phenotype including the renal function.

Vascular function is markedly affected by thyroid hormones that produce changes in vascular reactivity and endothelial function in thyroid dysfunction. Hypothyroidism is accompanied by a marked decrease in sensitivity to vasoconstrictors [11]. However, studies of changes in renal blood flow in the presence of hypothyroidism have produced conflicting results [26, 27]. Our study showed that the function of the peripheral artery and renal artery did not differ significantly after thyroxine replacement therapy. Different study design and analysis tools might lead to the conflicting results. More sophisticated studies are needed to resolve this conflict.

Our study had several limitations. Radiation causes significant kidney injury. Previous studies had showed that radiation could increase renal inflammation, oxidative stress, and kidney fibrosis $[28,29]$. Since radioactive iodine targets thyroid cells only, it is a safe and effective way to treat thyroid cancer [30]. As radioactive iodine is most concentrated in the thyroid gland, the toxic effect of radioiodine in the kidney will be minimal. In addition, our study was limited by its small population, which precluded a more reliable statistical analysis of the data. Our study design was that the same study population received testing before and after the therapy, as a result any confounding caused by the difference of individual characteristics might be minimal.

In conclusion, the GFR of hypothyroid patients increased significantly after thyroxine replacement. The change of GFR was significantly correlated with the changes of $\mathrm{fT}_{4}, \mathrm{CPK}$, myoglobulin and LVEF, after thyroxine replacement therapy. Our study suggested that TSH might not be a good clinical indicator to monitor hypothyroid phenotype including the renal function.

\section{Acknowledgment}

We are grateful for the grant from Chang Gung Memorial Hospital (CMRPG 270211E).

\section{References}

1 Song SH, Kwak IS, Lee DW, Kang YH, Seong EY, Park JS: The prevalence of low triiodothyronine according to the stage of chronic kidney disease in subjects with a normal thyroid-stimulating hormone. Nephrol Dial Transplant 2009;24:1534-1538.

2 Verhelst J, Berwaerts J, Marescau B, et al: Serum creatine, creatinine and other guanidine compounds in patients with thyroid dysfunction. Metabolism 1997; 46:10631067.

-3 Akcakoyun M, Kaya H, Kargin R, et al: Abnormal left ventricular longitudinal functional reserve assessed by exercise pulsed wave tissue Doppler imaging in patients with subclinical hypothyroidism. J Clin Endocrinol Metab 2009;94:2979-2983.

-4 Kreisman SH, Hennessey JV: Consistent reversible elevations of serum creatinine levels in severe hypothyroidism. Arch Intern Med 1999;159:79-82.
5 Hammami MM, Alsaihati B, Alahmari S, Alnuaim AA, Khan BA: Elevated serum creatinine level in thyroid cancer patients undergoing withdrawal of thyroxine therapy for radioiodine scan/treatment. Ann Saudi Med 1995;15:358-362.

6 6 Davis RG, Madsen KM, Fregly MJ, Tisher CC: Kidney structure in hypothyroidism. Am J Pathol 1983;113:41-49.

7 Claus T, Elitok S, Schmitt R, Luft FC, Kettritz R: Thyroid function and glomerular filtration - a potential for grave errors. Nephrol Dial Transplant 2005;20:1002-1003.

8 Villabona C, Sahun M, Roca M, et al: Blood volumes and renal function in overt and subclinical primary hypothyroidism. Am J Med Sci 1999;318:277-280.

-9 Kagiyama S, Tsuruta H, Tominaga M, Morishita K, Doi Y, Onoyama K: Minimal change nephritic syndrome and acute renal failure in a patient with aged onset insulin-dependent mellitus and autoimmune thyroiditis. Am J Nephrol 1999;19:369-372.
10 Kaptein EM, Wilcox RB, Nelson JC: Assessing thyroid hormone status in a patient with thyroid disease and renal failure: from theory to practice. Thyroid 2004;14:397-400.

11 Vargas F, Moreno JM, Rodríguez-Gómez I, et al: Vascular and renal function in experimental thyroid disorders. Eur J Endocrinol 2006;154:197-212.

12 Nakahama H, Sakaguchi K, Horita Y, et al: Treatment of severe hypothyroidism reduced serum creatinine levels in two chronic renal failure patients. Nephron 2001;88:264267.

13 Giampietro O, Clerico A, Buzzigoli G, Del Chicca MG, Boni C, Acrpi A: Detection of hypothyroid myopathy by measurements of various serum muscle markers - myoglobin, creatine kinase, lactate dehydrogenase and their isoenzymes. Horm Res 1984;19:232242.

14 Anwar S, Gibofsky A: Musculoskeletal manifestations of thyroid disease. Rheum Dis Clin North Am 2010;36:637-646. 
-15 Den Hollander JG, Wulkan RW, Mantel MJ, Berghout A: Correlation between severity of thyroid dysfunction and renal function. Clin Endocrinol 2005;62:423-427.

16 Lim VS: Thyroid function in patients with chronic renal failure. Am J Kidney Dis 2001; 38(suppl 1):S80-S84.

17 Bald M, Hauffa BP, Wingen A: Hypothyroidism mimicking chronic renal failure in reflux nephropathy. Arch Dis Child 2000;83: 251-252.

-18 Baajafer FS, Hammami MM, Mohamed GE: Prevalence and severity of hyponatremia and hypercreatininemia in short-term uncomplicated hypothyroidism. J Endocrinol Invest 1999;22:35-39.

19 Letizia C, Centanni M, Cesareo R, et al: Increased plasma levels of endothelin-1 in patients with hyperthyroidism. Metabolism 1995;44:1239-1242.
20 Strauer BE, Schulze W: Experimental hypothyroidism: depression of myocardial contractile function and hemodynamics and their reversibility by substitution with thyroid hormones. Basic Res Cardiol 1976;71: 624-644.

21 Danzi S, Klein I: Thyroid hormone and the cardiovascular system. Minerva Endocrinol 2004;29:139-150.

22 Pearce EN, Yang Q, Benjamin EJ, Aragam J Vasan RS: Thyroid function and left ventricular structure and function in the Framingham Heart Study. Thyroid 2010;20:369-373

23 Gerdes AM, Iervasi G: Thyroid replacement therapy and heart failure. Circulation 2010; 122:385-393.

24 Montenegro J, González O, Saracho R, Aguirre R, González O, Martínez I: Changes in renal function in primary hypothyroidism. Am J Kidney Dis 1996;27:195-198.

25 Iglesias P, Díez JJ: Thyroid dysfunction and kidney disease. Eur J Endocrinol 2009;160. 503-515.
26 Vargas F, Atucha NM, Sabio JM, Quesada T, Garcia-Estan J: Pressure-diuresis-natriuresis response in hyperthyroid and hypothyroid rats. Clin Sci (Lond) 1994;87:323-328.

$>27$ Gumieniak O, Perlstein TS, Hopkins PN, et al: Thyroid function and blood pressure homeostasis in euthyroid subjects. J Clin Endocrinol Metab 2004;89:3455-3461.

-28 Liu DG, Wang TM: Role of connective tissue growth factor in experimental radiation nephropathy in rats. Chin Med J 2008;121: 1925-1931.

29 Zhao W, Robbins ME: Inflammation and chronic oxidative stress in radiation-induced late normal tissue injury: therapeutic implications. Curr Med Chem 2009;16:130143.

30 Van Nostrand D, Wartofsky L: Radioiodine in the treatment of thyroid cancer. Endocrinol Metab Clin North Am 2007;36:807-822. 